\title{
Desarrollo y tendencias de la ingeniería biomédica en México
}

José de Jesús Agustín Flores Cuautle ${ }^{1}$

Citlalli Jessica Trujillo Romero ${ }^{*}$

Rigoberto Martínez Méndez ${ }^{3}$

Blanca Estela González Sánchez ${ }^{4}$

Ingrid Lizette Sánchez Carmona ${ }^{4}$

${ }^{1}$ CONACYT-Tecnológico Nacional de México, I. T. Orizaba

División de investigación en Ingeniería Médica, Instituto Nacional de Rehabilitación LGIl., México 3Universidad Autónoma del Estado de México, Facultad de Ingeniería. Cerro de Coatepec s/n, Ciudad Universitaria

4 Tecnológico Nacional de México, I. T. Orizaba

*Corresponding author: yoda34@hotmail.com 


\section{"Desarrollo y tendencias de la ingeniería biomédica en México"}

\section{Resumen}

La ingeniería biomédica una ciencia interdisciplinaria, a la cual se incorporan cada vez más estudiantes por medio de las diferentes instituciones que ofrecen programas de estudio relacionados con esta disciplina, con la finalidad de entender el rumbo que actualmente está tomando esta disciplina, es necesario conocer los principales programas de estudio que ofrecen las diversas instituciones, de la misma forma, finalmente se presenta una reflexión sobre los temas de actualidad relacionados con esta disciplina.

\section{Ingeniería Biomédica}

La Ingeniería Biomédica es un área del conocimiento multidisciplinaria, de acuerdo con el Imperial College London y a la IEEE Engineering in Medicine \& Biology Society ("Aims and scope," 2020; "Definition of Biomedical Engineering," 2020), la ingeniería biomédica es una disciplina que se enfoca al estudio de la evolución del conocimiento de la ingeniería, la biología y la medicina para hacer del mundo un lugar más saludable. Dicho objetivo se logra mediante actividades interdisciplinarias entre las cuales destacan las dos siguientes:

- La adquisición de conocimiento nuevo y entendimiento de los sistemas vivos mediante el uso innovador de técnicas experimentales y analíticas basadas en las ciencias de la ingeniería.

- El desarrollo de nuevos dispositivos, algoritmos, procesos y sistemas que promuevan la biología y la medicina y que a su vez mejoren la práctica médica y la prestación de los servicios de salud.

Para lograr los objetivos antes descritos se debe incluir no sólo la aplicación de los avances tecnológicos de la ingeniería al área médica, sino también a las ciencias básicas de la vida. Los retos de un ingeniero biomédico son utilizar su experiencia y conocimientos en la ingeniería, la medicina, la biología, la física, las matemáticas y las comunicaciones para tratar de resolver los problemas de salud que se presentan en el mundo. La Figura 1 muestra la interacción de las diferentes disciplinas de las que se sirve un Ingeniero biomédico para realizar su labor. Un ingeniero biomédico trabaja en una intersección entre la ciencia, la medicina y las matemáticas con la finalidad de resolver problemas médicos y biológicos; así como diseñar, desarrollar y administrar la tecnología en las unidades de salud (Bravo Hernandez, 2020; Voigt \& Magjarević, 2014).

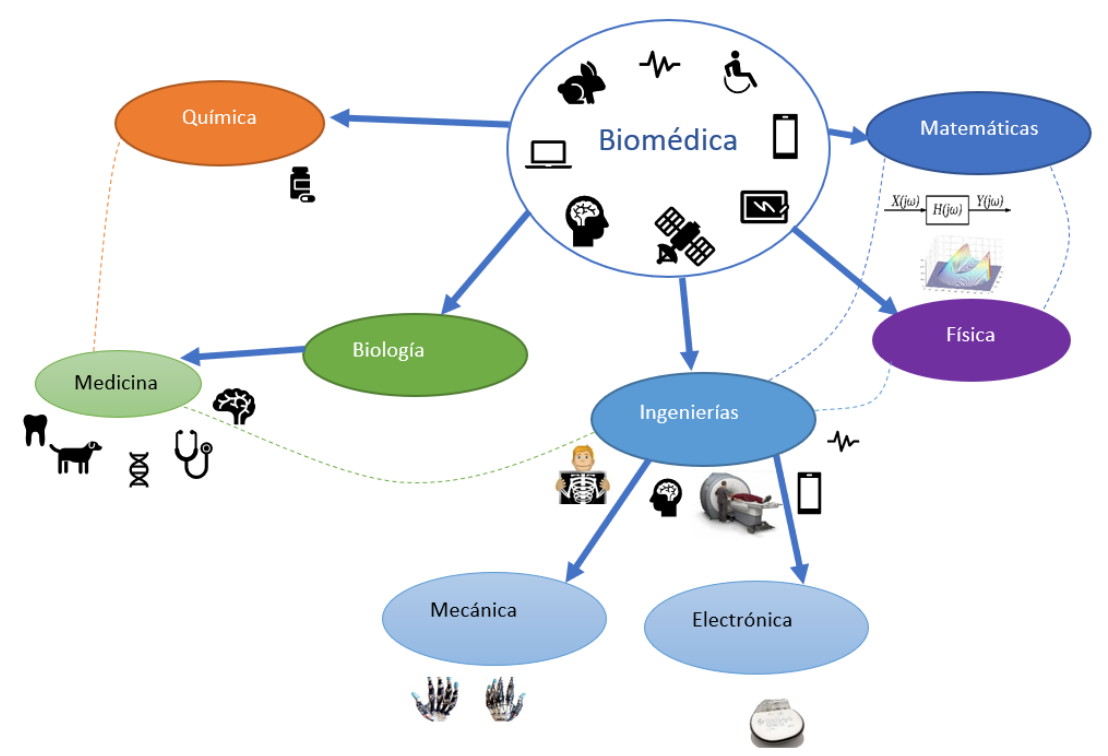

Fig. 1 La ingeniería biomédica y su interacción con otras disciplinas. 
Es importante resaltar que debido a la gran variedad de disciplinas que intervienen en la ingeniería biomédica, los ingenieros biomédicos pueden y aplican conocimientos de electricidad, electrónica, mecánica, química y todos aquellos relacionados con la ingeniería. Por lo tanto, no sólo aquellos con un título de ingeniería biomédica participan de esta disciplina. De manera general, todo aquel que tenga un título de ingeniería que le haya permitido obtener la experiencia en una o más de las áreas de investigación y aplicación de la ingeniería biomédica puede participar activamente en esta disciplina (Enderle, Bronzino, \& Blanchard, 2005).

\section{La ingeniería biomédica en el mundo}

Alrededor del mundo, la ingeniería biomédica es vista como una disciplina con un gran potencial y con una predicción de crecimiento importante. De acuerdo con el Wall Street Journal (Harris, 2017), en Estados Unidos se estima que el número de ingenieros biomédicos se incrementó en un $72 \%$ respecto al 2010. Por otro lado, estimaciones hechas por el departamento de empleo de los Estados Unidos prevén que la expectativa de crecimiento para esta disciplina es del $23 \%$, con un salario medio de 86,000 dólares americanos al año (Statistics, 2017). No sólo en Estados Unidos se dan este tipo de expectativas, en general las profesiones relacionadas con la salud tienen proyecciones de crecimiento elevadas. Por esta razón, no es de sorprenderse que las diversas instituciones educativas alrededor del mundo ofrezcan entre sus programas de estudio la carrera de ingeniería biomédica, no sólo a nivel licenciatura sino también a nivel posgrado.

Es notable el hecho de que la carrera de ingeniería biomédica es ofrecida no sólo por universidades reconocidas por sus programas en Ingeniería, sino que escuelas tales como la Escuela de Medicina John Hopkins también ofrecen este tipo de ingeniería tanto a nivel licenciatura como a nivel posgrado ("Johns Hopkins Biomedical Engineering Graduate," 2020; "Johns Hopkins Biomedical Engineering Undergraduate," 2020). Diversos estudios dan cuenta del incremento de instituciones académicas que ofrecen en sus planes de estudio programas relacionados con la ingeniería Biomédica. Tan sólo en Estados Unidos se pasó de 102 universidades en el año 2002 a 119 en 2006, en tanto que alrededor del mundo se reportan 10,453 instituciones académicas ofreciendo este tipo de programas ("Biomedical engineers data by country," 2016).

De igual manera, alrededor del mundo existen sociedades civiles dedicadas a la promoción y desarrollo de la ingeniería biomédica, entre las principales podemos mencionar la Biomedical Engineering Society ("Biomedical Engineering society," 2020), el American Institute for Medical and Biological Engineering ("American Institute for medical and biological engineering," 2020), la Engineering in Medicine \& Biology Society ("Engineering in medicine and biology society," 2020). Estas asociaciones agrupan cerca de 500,000 miembros alrededor del mundo; además, tienen presencia en más de 160 países. La Tabla 1 muestra algunas de las principales sociedades civiles dedicadas a la promoción de la ingeniería biomédica; así como el número de miembros con los que cuentan.

Tabla 1 Principales sociedades civiles enfocadas a la promoción de la ingeniería biomédica

\begin{tabular}{|l|l|}
\hline Nombre de la Sociedad & $\begin{array}{l}\text { Número } \\
\text { miembros }\end{array}$ \\
\hline $\begin{array}{l}\text { Biomedical Engineering Society (BMES) ("Biomedical Engineering society," } \\
2020)\end{array}$ & 7,000 \\
\hline $\begin{array}{l}\text { The American Institute for Medical and Biological Engineering (AIMBE) } \\
\text { ("American Institute for medical and biological engineering," 2020) }\end{array}$ & 50,000 \\
\hline $\begin{array}{l}\text { Engineering in Medicine and Biology Society (EMBS), ("Engineering in } \\
\text { medicine and biology society," 2020) }\end{array}$ & 9,100 \\
\hline $\begin{array}{l}\text { The Institute of Electrical and Electronic Engineers (IEEE).("Aims and scope," } \\
2020)\end{array}$ & 425,000 \\
\hline
\end{tabular}




\section{Breve historia de la Ingeniería biomédica en México}

Existen diversas definiciones de ingeniería biomédica, dentro de estas, la mayoría hace uso de palabras y frases tales como: "aplicación de ciencias exactas", "solución de problemas del sector salud", "biomedicina", "bioelectrónica", etc. ("Ingenieria Biomedica," 2016; "Ingeniería Biomédica," 2016; "Universidad de Barcelona," 2016). Con base en lo anterior, es difícil encontrar el momento exacto en el cual surge la ingeniería biomédica en México. Algunos autores hablan del nacimiento de la ingeniería biomédica basados en la creación del Instituto de Salubridad y Enfermedades Tropicales (ISTE) en 1939 (Mendez \& Leehan, 2003; Perez-Tamayo, 2004). Sin embargo, la mayoría de quienes han escrito sobre esta disciplina, coinciden en dos momentos claves para la misma:

1) Creación del Instituto Nacional de Cardiología, lugar en el cual científicos de la talla de Norbert Wiener, Albert Grass y Arturo Rosenblueth, entre otros, comienzan labores de investigación relacionadas no sólo con la fisiología, sino con el desarrollo de utensilios y equipos que facilitaran sus labores de investigación (Glantz, 2015; Pineda Romero \& González Guerrero, 2001; Voigt \& Magjarević, 2014).

2) El surgimiento del primer programa académico de Maestría en Ciencias en Bioelectrónica en el recién creado CINVESTAV hacia 1974 por el Dr. Joaquín Remolina. Este posgrado nace como una escisión del Departamento de Farmacología y Toxicología (Cadena \& Azpiroz, 2003; Muñoz Martinez, 2005)

Hasta ese momento sólo se contaba con centros de investigación y laboratorios y es hasta 1973 cuando la Universidad Iberoamericana crea el primer programa de licenciatura en Ingeniería Biomédica ("Sociedad Mexicana de Ingeniería Biomédica," 2016). Otras universidades como la Universidad Nacional Autónoma de México cuentan con su propia historia acerca de cómo surgen los programas de ingeniería biomédica en las mismas. El Instituto de Investigaciones Biomédicas (1969) es reconocido como el organismo que dirige los destinos de los planes y programas de estudio en ingeniería biomédica y que tiene como antecedente directo el Laboratorio de Estudios Médicos y Biológicos (1941) ("Instituto de Investigaciones Biomedicas," 2016). En 1973 se crea el proyecto académico de licenciatura, Maestría y Doctorado en investigación biomédica básica de la Universidad Autónoma de México (Kaethe, 2016).

Respecto a los esfuerzos fuera del ámbito netamente académico, podemos mencionar la fundación de la Sociedad Mexicana de Ingeniería Biomédica hacia 1978 ("Sociedad Mexicana de Ingeniería Biomédica," 2016), la cual se define como "una Asociación civil no lucrativa con intereses profesionales, académicos y científicos". Al año siguiente se publica La Revista Mexicana de Ingeniería Biomédica con la finalidad de dar difusión a los trabajos realizados en esta área ("Sociedad Mexicana de Ingeniería Biomédica," 2016). 


\section{La Ingeniería Biomédica en México}

En lo que respecta a el ámbito formativo, la encuesta global de estudios sobre ingeniería biomédica 2016 reporta 13 instituciones en México que ofrecen programas de estudio relacionados con esta disciplina ("Biomedical engineers data by country," 2016). Por otro lado, la Sociedad Mexicana de Ingeniería Biomédica reporta 45 instituciones contando los diferentes campus localizados en 21 estados de la República Mexicana (Educacion, 2020). En la figura 2 se muestran las diferentes áreas en las que los investigadores nacionales obtuvieron su doctorado, separadas por área y por año. En dicha gráfica se aprecia un incremento gradual de investigadores, tanto en el área de la biomédica como en la bioingeniería, incrementándose en forma exponencial en el último año que abarca este estudio.

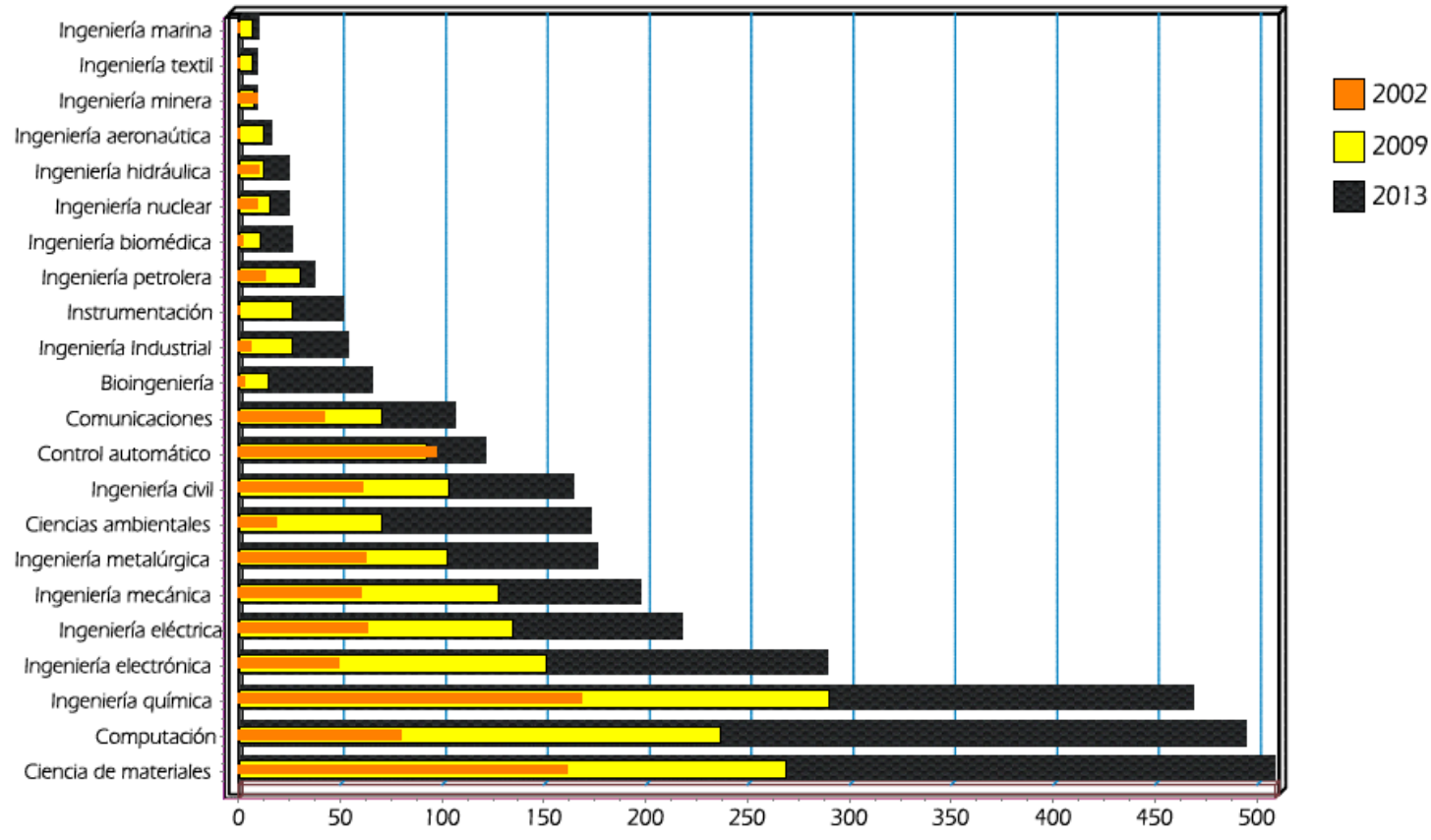

Figura 2. Distribución de las áreas donde los investigadores obtuvieron su doctorado (Perez Angon, 2014)

\section{Programas de estudio de ingeniería biomédica por estados}

En lo referente a los estudios de posgrado específicamente en el área de ingeniería biomédica, se deben mencionar instituciones tales como el Instituto Tecnológico de Tijuana, la Universidad Nacional Autónoma de México, la Universidad Autónoma Metropolitana, la Universidad Autónoma de Pachuca y la Universidad Iberoamericana. Sin embargo, si tomamos en cuenta carreras que se encuentran estrechamente relacionadas con la Ingeniería Biomédica, tales como Física Médica, Bioelectrónica, Instrumentación, etc. el número de posgrados ofrecidos en México se incrementa exponencialmente. De acuerdo con la Sociedad Mexicana de Ingeniería Biomédica, actualmente se encuentran registrados en el país más de 50 programas de ingeniería biomédica en México ("Sociedad Mexicana de Ingeniería Biomédica," 2016).

La Tabla 2 muestra algunos de los programas de licenciatura que se ofertan en el país; por otro lado, la Tabla 3 muestra los programas de posgrado. Así mismo, se muestran las áreas del Sistema Nacional de Investigadores (SNI) correspondientes a los diferentes programas de estudio. 
Tabla 2 licenciaturas ofrecidas en México relacionadas con la Ingeniería Biomédica

\begin{tabular}{|c|c|c|c|}
\hline Programa & Institución & Entidad & Área SNI \\
\hline $\begin{array}{l}\text { Licenciatura } \\
\text { ingeniería } \\
\text { biotecnología }\end{array}$ & $\begin{array}{l}\text { Universidad } \\
\text { popular } \\
\text { autónoma del estado de } \\
\text { puebla }\end{array}$ & Puebla & Biología \\
\hline $\begin{array}{l}\text { Licenciatura } \\
\text { biotecnología }\end{array}$ & $\begin{array}{l}\text { Benemérita universidad } \\
\text { autónoma de Puebla }\end{array}$ & Puebla & $\begin{array}{l}\text { Biología, } \\
\text { ingeniería } \\
\text { tecnología }\end{array}$ \\
\hline $\begin{array}{l}\text { Licenciatura } \\
\text { biotecnología }\end{array}$ & $\begin{array}{l}\text { Universidad autónoma de } \\
\text { Aguascalientes }\end{array}$ & Aguascalientes & $\begin{array}{l}\text { Química } \\
\text { Biología }\end{array}$ \\
\hline $\begin{array}{l}\text { Licenciatura en } \\
\text { ingeniería biomédica }\end{array}$ & $\begin{array}{l}\text { Universidad La Salle de } \\
\text { México }\end{array}$ & México & \\
\hline $\begin{array}{l}\text { Licenciatura en } \\
\text { ingeniería biomédica }\end{array}$ & Universidad de Guanajuato & Guanajuato & Medicina \\
\hline $\begin{array}{l}\text { Licenciatura en } \\
\text { ingeniería biomédica }\end{array}$ & $\begin{array}{ll}\text { Universidad } & \text { Autónoma } \\
\text { Metropolitana } & \end{array}$ & CDMX & Medicina \\
\hline Ingeniería biomédica & Universidad de Guadalajara & Guadalajara & $\begin{array}{l}\text { Ciencias exactas e } \\
\text { ingenierías }\end{array}$ \\
\hline Ingeniería biomédica & $\begin{array}{l}\text { Universidad Politécnica de } \\
\text { Pachuca }\end{array}$ & Hidalgo & $\begin{array}{l}\text { Ciencias exactas e } \\
\text { ingenierías }\end{array}$ \\
\hline $\begin{array}{l}\text { Licenciatura en } \\
\text { investigación biomédica }\end{array}$ & UNAM & México & \\
\hline Ingeniería biomédica & Universidad iberoamericana & México & \\
\hline $\begin{array}{l}\text { Licenciatura } \\
\text { biomedicina }\end{array}$ & BUAP & Puebla & $\begin{array}{l}\text { Ciencias naturales } \\
\text { y de la salud }\end{array}$ \\
\hline Ingeniería bioelectrónica & Universidad panamericana & Aguascalientes & \\
\hline Ingeniería en biomédica & Instituto politécnico nacional & CDMX & Medicina \\
\hline Ingeniería biomédica & Universidad Modelo & Yucatán & \\
\hline
\end{tabular}


Tabla 3 Programas de posgrado ofrecidos en México relacionadas con la Ingeniería Biomédica

\begin{tabular}{|c|c|c|c|}
\hline Programa & Institución & Entidad & Área SNI \\
\hline $\begin{array}{l}\text { Maestría En Ciencias En } \\
\text { Biomedicina }\end{array}$ & $\begin{array}{l}\text { Universidad Autónoma De } \\
\text { Querétaro }\end{array}$ & Querétaro & $\begin{array}{l}\text { Medicina Y Ciencias } \\
\text { De La Salud }\end{array}$ \\
\hline $\begin{array}{l}\text { Maestría En Ciencias } \\
\text { Biomédicas }\end{array}$ & $\begin{array}{l}\text { Universidad Autónoma De } \\
\text { Sinaloa }\end{array}$ & Sinaloa & $\begin{array}{l}\text { Medicina Y Ciencias } \\
\text { De La Salud }\end{array}$ \\
\hline Maestría En Ciencias & Universidad Autónoma Del & Hidalgo & Medicina Y Ciencias \\
\hline $\begin{array}{l}\text { Biomédicas } \\
\text { Salud }\end{array}$ & Estado De Hidalgo & & De La Salud \\
\hline $\begin{array}{l}\text { Maestría En Ciencias En } \\
\text { Biomedicina Molecular }\end{array}$ & $\begin{array}{l}\text { Centro De Investigación Y De } \\
\text { Estudios Avanzados Del } \\
\text { Instituto Politécnico Nacional }\end{array}$ & $\begin{array}{l}\text { Ciudad De } \\
\text { México }\end{array}$ & $\begin{array}{l}\text { Medicina Y Ciencias } \\
\text { De La Salud }\end{array}$ \\
\hline $\begin{array}{l}\text { Maestría En Ingeniería } \\
\text { Biomédica }\end{array}$ & $\begin{array}{l}\text { Universidad Autónoma } \\
\text { Metropolitana }\end{array}$ & $\begin{array}{l}\text { Ciudad De } \\
\text { México }\end{array}$ & Ingenierías \\
\hline $\begin{array}{l}\text { Maestría En Ciencias } \\
\text { Biomédicas }\end{array}$ & $\begin{array}{l}\text { Universidad Juárez } \\
\text { Autónoma De Tabasco }\end{array}$ & Tabasco & Biología Y Química \\
\hline $\begin{array}{l}\text { Maestría En Ciencias En } \\
\text { Biomedicina Y } \\
\text { Biotecnología Molecular }\end{array}$ & Instituto Politécnico Nacional & $\begin{array}{l}\text { Ciudad } \\
\text { México }\end{array}$ & $\begin{array}{l}\text { Medicina Y Ciencias } \\
\text { De La Salud }\end{array}$ \\
\hline $\begin{array}{l}\text { Maestría En Biomedicina } \\
\text { Molecular }\end{array}$ & Instituto Politécnico Nacional & $\begin{array}{l}\text { Ciudad De } \\
\text { México }\end{array}$ & $\begin{array}{l}\text { Medicina Y Ciencias } \\
\text { De La Salud }\end{array}$ \\
\hline $\begin{array}{l}\text { Maestría En Ciencias } \\
\text { Biomédicas }\end{array}$ & $\begin{array}{lll}\text { Instituto Tecnológico } & \text { Y } & \text { De } \\
\text { Estudios } & \text { Superiores } & \text { De } \\
\text { Monterrey } & & \end{array}$ & Nuevo León & $\begin{array}{l}\text { Medicina Y Ciencias } \\
\text { De La Salud }\end{array}$ \\
\hline $\begin{array}{l}\text { Maestría En Ciencias } \\
\text { Biomédicas }\end{array}$ & $\begin{array}{l}\text { Universidad Autónoma De } \\
\text { Guerrero }\end{array}$ & Guerrero & $\begin{array}{l}\text { Medicina Y Ciencias } \\
\text { De La Salud }\end{array}$ \\
\hline $\begin{array}{l}\text { Maestría } \\
\text { Biotecnología }\end{array}$ & $\begin{array}{l}\text { Universidad Autónoma De } \\
\text { Sinaloa }\end{array}$ & Sinaloa & $\begin{array}{l}\text { Biotecnología } \\
\text { Ciencias } \\
\text { Agropecuarias }\end{array}$ \\
\hline $\begin{array}{l}\text { Maestría } \\
\text { Biotecnología }\end{array}$ & $\begin{array}{l}\text { Universidad Autónoma Del } \\
\text { Estado De Morelos }\end{array}$ & Morelos & $\begin{array}{l}\text { Biotecnología } \\
\text { Ciencias } \\
\text { Agropecuarias }\end{array}$ \\
\hline $\begin{array}{l}\text { Maestría } \\
\text { Biotecnología }\end{array}$ & $\begin{array}{l}\text { Universidad } \\
\text { Metropolitana }\end{array}$ & $\begin{array}{l}\text { Ciudad } \\
\text { México }\end{array}$ & $\begin{array}{l}\text { Biotecnología } \\
\text { Ciencias } \\
\text { Agropecuarias }\end{array}$ \\
\hline $\begin{array}{l}\text { Maestría } \\
\text { Biotecnología }\end{array}$ & Universidad Del Papaloapan & Oaxaca & $\begin{array}{l}\text { Biotecnología } \\
\text { Ciencias } \\
\text { Agropecuarias }\end{array}$ \\
\hline $\begin{array}{l}\text { Maestría En Ciencias En } \\
\text { Biotecnología }\end{array}$ & $\begin{array}{l}\text { Universidad Politécnica Del } \\
\text { Estado De Morelos }\end{array}$ & Morelos & $\begin{array}{l}\text { Biotecnología } \\
\text { Ciencias } \\
\text { Agropecuarias }\end{array}$ \\
\hline $\begin{array}{l}\text { Maestría } \\
\text { Biotecnología }\end{array}$ & $\begin{array}{ll}\text { Universidad } & \text { Popular } \\
\text { Autónoma Del } & \text { Estado De } \\
\text { Puebla, A.C. } & \end{array}$ & Puebla & $\begin{array}{l}\text { Biotecnología } \\
\text { Ciencias } \\
\text { Agropecuarias }\end{array}$ \\
\hline $\begin{array}{l}\text { Maestría } \\
\text { Biotecnología }\end{array}$ & $\begin{array}{l}\text { Universidad Autónoma De } \\
\text { Chihuahua }\end{array}$ & Chihuahua & $\begin{array}{l}\text { Biotecnología } \\
\text { Ciencias } \\
\text { Agropecuarias }\end{array}$ \\
\hline $\begin{array}{l}\text { Maestría En Ciencias Con } \\
\text { Especialidad } \\
\text { Biotecnología }\end{array}$ & $\begin{array}{lll}\text { Instituto Tecnológico } & \text { Y } & \text { De } \\
\text { Estudios } & \text { Superiores } & \text { De } \\
\text { Monterrey } & & \end{array}$ & Nuevo León & $\begin{array}{l}\text { Biotecnología } \\
\text { Ciencias } \\
\text { Agropecuarias }\end{array}$ \\
\hline
\end{tabular}




\section{Tendencias de los planes de estudio}

Con la finalidad de realizar un mejor análisis respecto a las asignaturas más representativas relacionadas con los planes de estudio de la licenciatura de Ingeniería Biomédica, las asignaturas se agruparon en 4 grandes áreas: básica, formativa, investigación y terminación formal. Es importante mencionar que, de acuerdo con los planes de estudio, el nombre de las asignaturas puede diferir entre una y otra institución. Sin embargo, en esencia las materias mencionadas tratan los mismos tópicos.

Tabla 4 Asignaturas representativas de la carrera de Ingeniería Biomédica Elaboración propia Con datos de ("Sociedad Mexicana de Ingeniería Biomédica," 2016)

\begin{tabular}{|c|c|c|c|c|c|c|c|}
\hline INSTITUCION & $\begin{array}{l}\text { UNIV. } \\
\text { POLI. DE } \\
\text { CHIAPAS }\end{array}$ & $\begin{array}{l}\text { IP } \\
\text { N }\end{array}$ & $\begin{array}{l}\mathbf{U} \\
\mathbf{N} \\
\mathbf{A} \\
\mathbf{M}\end{array}$ & $\begin{array}{l}\text { UNIV, } \\
\text { AUTÓNO } \\
\text { MA } \\
\text { METROP } \\
\text { OLITANA }\end{array}$ & $\begin{array}{l}\text { UNIV } \\
\text { AUTO. } \\
\text { EDO. } \\
\text { MEX. }\end{array}$ & $\begin{array}{l}\text { UNIV. } \\
\text { AUTO. } \\
\text { GUADALAJ } \\
\text { ARA }\end{array}$ & $\begin{array}{l}\text { UNIV. POLI. } \\
\text { BICENTEN } \\
\text { ARIO }\end{array}$ \\
\hline \multicolumn{8}{|l|}{ Asignatura } \\
\hline Matemáticas & $X$ & $X$ & $X$ & $X$ & $X$ & $X$ & $X$ \\
\hline $\begin{array}{l}\text { Biología } \\
\text { Anatomía }\end{array}$ & $\mathrm{x}$ & $X$ & $X$ & $\mathrm{X}$ & $\mathrm{X}$ & $X$ & $X$ \\
\hline $\begin{array}{l}\text { Instrumentació } \\
\text { n Biomédica }\end{array}$ & $\mathrm{X}$ & & $X$ & & & $X$ & $X$ \\
\hline $\begin{array}{l}\text { Electrónica } \\
\text { Analógica }\end{array}$ & $x$ & & & & $\mathrm{X}$ & $X$ & $X$ \\
\hline $\begin{array}{ll}\text { Sensores } & \text { Y } \\
\text { Actuadores } & \text { // } \\
\text { Biomédicos } & \end{array}$ & $X$ & & & & & & $X$ \\
\hline $\begin{array}{ll}\text { Señales } & \text { Y } \\
\text { Sistemas } & \end{array}$ & & & & $\mathrm{X}$ & & $\mathrm{X}$ & \\
\hline $\begin{array}{l}\text { Procesamiento } \\
\text { Digital de } \\
\text { Señales }\end{array}$ & & & & & & $\mathrm{X}$ & \\
\hline
\end{tabular}

Derivado de la Tabla 4 se aprecia que áreas tales como: Rehabilitación, biomateriales y física médica son prácticamente ignoradas en dichos planes de estudio a pesar de ser áreas claves para el desarrollo de la Ingeniería. La Tabla 4 muestra que los planes de estudio no siguen las tendencias mundiales en cuanto a nuevas áreas de investigación en Ingeniería Biomédica. Una búsqueda en una base de datos ("Elsevier Journals," 2020) arroja un promedio de 800,000 artículos en los últimos 5 años, cuando se coloca como palabra de búsqueda la frase "Ingeniería Biomédica", de esta búsqueda términos como biomateriales (30000), rehabilitación (100000), háptica (32000) Robótica (12000) son palabras comunes en esta búsqueda y como se ha mencionado ya, apenas han sido tomas en cuenta en el desarrollo de los planes y programas de estudio. 


\section{Una mirada más allá del mundo académico.}

En México se creó el Centro Nacional de Excelencia Tecnológica en Salud (CENETEC) cuya misión es cubrir las necesidades de gestión y evaluación de tecnologías lo cual es uno de los principales propósitos de la Ingeniería Biomédica. EI CENETEC incorpora dentro de su organigrama una Dirección de Ingeniería Biomédica cuya principal función es "atender los temas relacionados a la planeación y gestión de equipo médico, a través de la coordinación de los esfuerzos sectoriales e intersectoriales y promoviendo el desarrollo de las áreas de Ingeniería Biomédica" ("Centro Nacional de Excelencia Tecnológica en Salud ", 2020). En el año 2008 se propone la creación de los Centros estatales de Ingeniería Biomédica (CEDIB), cuya misión principal es apoyar a la toma de decisiones y el uso racional de los recursos dentro del Sistema Nacional de Salud en México. Sin embargo, a pesar de ser una iniciativa de gran utilidad en el área de la Ingeniería Biomédica este tema cayo en el olvido en tanto que en 2009 el CENETEC pasa a ser colaborador de la organización Panamericana de la Salud.

\section{Retos}

Es claro que el número de investigadores en el área de ingeniería biomédica se ha incrementado en los últimos años y esta tendencia continua al alza. Sin embargo, como en muchas otras áreas de investigación, la mayor parte de los profesores se caracteriza por tener una trayectoria netamente académica sin contar con experiencia alguna en el ámbito hospitalario, por ejemplo, que es uno de los sectores primordiales para esta área de estudio. Derivado de lo anterior, es claro que, como en muchas otras disciplinas, falta pasar del sector académico al sector productivo. Por otro lado, se debe tomar en cuenta también la falta de inversión en el sector salud, especialmente en el sector público, con todas las consecuencias que esto conlleva.

Por lo tanto, es necesario integrar a los planes y programas de estudio las líneas de investigación emergente. Esto con el fin de proporcionar una formación más amplia, integral y actual a los estudiantes de este tipo de áreas. De esta forma, el egresado no sólo tendrá los conocimientos básicos para cumplir a cabalidad su labor; sino al mismo tiempo tendrá las posibilidades de familiarizarse inmediatamente con las tecnologías que se están desarrollando en este momento y que serán de uso común en años venideros. Aunado a lo anterior, se hace presente la necesidad de atender los problemas crónico-degenerativos que se derivan del aumento de la edad en la población. Por lo tanto, es necesario atender los padecimientos propios de las personas en edad avanzada. A este respecto, tecnologías como wearable sensors, dispositivos móviles; es decir, sistemas embebidos que permiten no sólo el monitoreo continuo de pacientes, sino que al mismo tiempo facilitan el desarrollo de equipos de uso específico con poca instrumentación adicional es cada vez más frecuente. Sobre todo, en el monitoreo de padecimientos crónicos tal como la diabetes y la hipertensión, problemas que afectan de sobremanera a la población mexicana. La tendencia hacia el uso del $3 D$ printing no sólo como herramienta para construcción de prototipos, sino para la fabricación de férulas, prótesis, e incluso scafolds es un área que debe de fomentarse desde la academia para aprovechar las ventajas de esta tecnología y que debe de ir de la mano con el estudio de materiales. La Ingeniería Biomédica ha presentado grandes avances durante los últimos años; sin embargo, aún es necesario seguir implementando cambios para potenciar la resolución de problemas reales que se tienen en el sector salud de nuestro país. 


\section{Bibliografía}

Aims and scope. (2020). Retrieved from

https://ieeexplore.ieee.org/xpl/aboutJournal.jsp?punumber=51

American Institute for medical and biological engineering. (2020). Retrieved from https://aimbe.org/

Biomedical Engineering society. (2020). Retrieved from Biomedical Engineering society

Biomedical engineers data by country. (2016). Global health observatory data repository. Retrieved from http://apps.who.int/gho/data/view.main.BIOENGHWF8v

Bravo Hernandez, H. (2020). Dispositivos medicos, el rostro visible de la tecnologia de la salud. Retrieved from https://www.sectei.cdmx.gob.mx/comunicacion/nota/dispositivosmedicos-el-rostro-visible-de-la-tecnologia-de-lasalud?fbclid=IwAR2cdcvIEqGBxeo2hkKmM2kuEdHWmaVHsDMk3VDNRuMJuXh11D8pijtiF $\mathrm{Mg}$

Cadena, M., \& Azpiroz, J. (2003). Overview of the Biomedical Engineering History in Mexico: A Personal Point of View (Vol. 4).

Centro Nacional de Excelencia Tecnológica en Salud (2020). Retrieved from Centro Nacional de Excelencia Tecnológica en Salud

Definition of Biomedical Engineering. (2020). Retrieved from http://www3.imperial.ac.uk/pls/portallive/docs/1/51182.PDF

Educacion, C. d. (2020). IES Totales en Mexico. Retrieved from http://ies.somib.org.mx/ Enderle, J. D., Bronzino, J. D., \& Blanchard, S. M. (2005). Introduction to Biomedical Engineering: Elsevier Academic Press.

Engineering in medicine and biology society. (2020). Retrieved from https://www.embs.org/about-embs/

Glantz, R. G. (2015). Entre la experimentación y los modelos abstractos. Breve historia de vida de Arturo Rosenblueth (1900-1970). Antropología. Boletín Oficial del INAH, 99(2015).

Harris, R. (2017). Th breakdown in biomedical reseach. Retrieved from https://www.wsj.com/articles/the-breakdown-in-biomedical-research-1491576749

Ingenieria Biomedica. (2016). Retrieved from http://www.ingenieriabiomedica.org/\#!queesingenieriabiomedica/ch6q

Ingeniería Biomédica. (2016). Retrieved from http://www.ipn.mx/educacionsuperior/Paginas/Ing-Biomedica.aspx

Instituto de Investigaciones Biomedicas. (2016). Retrieved from http://www.biomedicas.unam.mx/acerca-del-instituto/quienes-somos/

Johns Hopkins Biomedical Engineering Graduate. (2020). Retrieved from https://www.bme.jhu.edu/graduate/overview/

Johns Hopkins Biomedical Engineering Undergraduate. (2020). Retrieved from https://www.bme.jhu.edu/undergraduate/objectives-outcomes/

Kaethe, W. (2016). La formación de investigadores en el proyecto academico de licenciatura, maestria y doctorado en investigacion biomedica basica. In U. Instituto de Investigaciones Biomédicas (Ed.).

Mendez, M. C., \& Leehan, J. A. (2003, 17-21 Sept. 2003). Overview of the biomedical engineering history in Mexico: a personal point of view. Paper presented at the Engineering in Medicine and Biology Society, 2003. Proceedings of the 25th Annual International Conference of the IEEE. 
Muñoz Martinez, E. J. (2005). Biografias de personajes ilustres, Joaquin Remolina Lopez. In G. Viesca Lopez (Ed.), Ciencia y tecnologia en Mexico en el siglo XXI (Vol. IV). Mexico: Academia Mexicana de Ciencias.

Perez-Tamayo, R. (2004). La investigación biomédica en México. Gaceta Médica de México, 140(S1), 6.

Perez Angon, M. A. (2014). Atlas de la Ciencia Mexicana 2014 Vol. 1.

Pineda Romero, M. M., \& González Guerrero, K. (2001). REFLEXIONES SOBRE BIOÉTICA E INGENIERÍA BIOMÉDICA: INTRÍNSECA COMPLEMENTARIEDAD. Revista Latinoamericana de Bioética, 10(2).

Sociedad Mexicana de Ingeniería Biomédica. (2016). Retrieved from http://somib.org.mx/

Statistics, B. o. L. (2017). Occupational Outlook Handbook, 2016-17 Edition, Biomedical Engineers. Retrieved from https://www.bls.gov/ooh/architecture-and-engineering/biomedicalengineers.htm

Universidad de Barcelona. (2016). Retrieved from http://www.ub.edu/web/ub/es/estudis/oferta_formativa/graus/fitxa/I/G1074/index.html

Voigt, H., \& Magjarević, R. (2014). Ingeniería Biomédica en México (Una Visión Sucinta). In H. Voigt \& R. Magjarevic (Eds.), Launching IFMBE into the 21st Century: 50 Years and Counting (pp. 201-203). Berlin, Heidelberg: Springer Berlin Heidelberg.

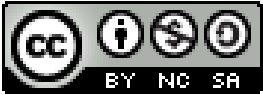

Esta obra está bajo una licencia de Creative Commons Reconocimiento-NoComercial-CompartirIgual 2.5 México. 